\title{
On the correctness of one model of the Stefan-Type filtration theory
}

\author{
Mukhambetzhanov S.T., Zhanuzakova \\ D.T. 050023, Almaty, Kazakhfilm, 12-6 \\ Kazakhstan
}

\begin{abstract}
In this paper a mathematical model of filtration theory with phase transitions is investigated. When using surface-active substances (surfactants) for the development of oil and gas fields in the reservoir occur sorption processes at the interfaces of individual phases (surfactants and oil, or surfactants and soil). In real processes, a finite time is required for achievement equilibrium. Therefore considering the mathematical model was called the mathematical model with phase relaxation. The solvability of the mathematical model, the limiting transition in relaxation time are investigated. It is proved that in the limiting case, the original problem is a problem of Stefan type.
\end{abstract}

Keywords. Sorption, adsorption, surfactant, relaxation time, mass transfer processes, numerical experiments.

\section{INTRODUCTION}

This paper is a logical continuation of studying the mathematical model presented in [1]. However, between phases, many authors believe that either Henry's law or Langmuir's law is fulfilled. Based on the results of [2], below we assume that there is some characteristic relaxation time for achieving equilibrium between the phases:

$$
\frac{\partial s}{\partial t}=\frac{1}{\tau}(H(c)-s)
$$

where $\tau$ positive constant and called relaxation time. Then the concentration of surfactant $c(x, t)$ is a solution to the following equation:

$$
m \cdot \frac{\partial c}{\partial t}=D \cdot \Delta c-v \cdot \nabla c-\frac{\partial s}{\partial t},
$$

where $m, D$ - positive constants, $v$ - rate of fluid filtration in a porous medium, function $\mathrm{H}(\mathrm{c})=1 \quad$ if $\quad \mathrm{c}(x, t)>c_{*}, \mathrm{H}(\mathrm{c})=0 \quad$ if $\mathrm{c}(x, t)<c_{*}$ and in the case of equilibrium processes $s=H(c)$. Then the system (1), (2) reduces to the well-known Stefan problem. Similar mathematical models were investigated in $[1,2,7]$.

\section{FORMULATION OF THE PROBLEM}

Let $\Omega$ - is a bounded domain in $R^{m}$ with a sufficiently smooth border $\Gamma, Q_{T}=$ $\Omega x(0, T), \quad \Gamma_{T}=\Gamma x(0, T)$. Required to find functions $\quad c(x, t), s(x, t) \quad$ (surfactant concentrations in liquid and solid phases), defined in the area $Q_{T}$, satisfying equations (1), (2) and initial conditions

$$
\begin{array}{r}
c(x, 0)= \\
c_{0}(x), \quad s(x, 0)=s_{0}(x), \quad x \in \Omega
\end{array}
$$

and one of the boundary conditions

$$
c(x, t)=c_{\Gamma}(x, t), \quad(x, t) \in \Gamma_{T}
$$

$$
\frac{\partial c}{\partial n}-v \cdot c(x, t)=c_{\Gamma}(x, t), \quad(x, t) \in \Gamma_{T}
$$

Here $\mathrm{n}$ - internal normal vector to $\mathrm{S}$. 
Further, under task I is understood as task (1)-(4), and task II is understood as task II (1)-(3), (5).

Definition 1. The solution of problem I (problem II) is a pair of functions $\{c, s\}$ such that:

1. $c \in W_{q}^{2,1}\left(Q_{T}\right), \quad 1<q<\infty, \quad s_{t}$, $s \in L_{\infty}\left(Q_{T}\right)$

2. Equations (1), (2) are performed almost everywhere (a. e.) in $Q_{T}$;

3. Initial and boundary conditions for $c(x, t)$ are accepted in the sense of traces of functions from the indicated classes, for $s$ the initial condition is taken as follows $\| s(x, t)-$ $s_{0} \|_{\infty, \Omega} \rightarrow 0$ at $t \rightarrow 0$.

Designations norms and spaces of functions coincide with the notation in [2].

\section{AUXILIARY SENTENCES}

Lemma 1. Let $u \in W_{p}^{1}(Q), Q$ - is a bounded domain in $R^{k}, \quad p>1$, $A_{\varepsilon}=\{x \in Q|| u(x) \mid \leq \varepsilon\}$. Then $\nabla u=0$ a. e. in $A_{0}$.

Lemma 2. Let $Q$ - is a bounded domain in $R^{k}, v_{n}, v, g \in L_{p}(Q), p>1, \forall x \in Q \backslash A$, $\varliminf_{n \rightarrow \infty} v_{n}(x)=f(x)$ and $\forall n \in N,\left|v_{n}(x)\right| \leq g(x)$, where mes $A=0 ; v_{n} \rightarrow v$ weakly in $L_{p}(Q)$. Then $v \geq f$ a. e. in $Q$.

From Lemma 1 it follows that in the domain $\quad E_{c}=\left\{(x, t) \in Q_{T} \mid c(x, t)=0\right\}$ equalities are performed: $c_{t}=\Delta c=0$. Then from equations (1), (2) are displayed: $H(c(x, t))=s(x, t)$ for a. e. $(x, t) \in E_{c}$. From the definition of the function $\mathrm{H}$ (c), in particular, it follows that $0 \leq s(x, t) \leq 1$ for a. e. $(x, t) \in E_{c}$.

3. The correctness of the mathematical model. Replacement of independent variables and sought functions is done:

$$
\begin{gathered}
t^{\prime}=\frac{t}{\tau}, \quad x^{\prime}=x \sqrt{m / \tau D}, \quad s^{\prime}= \\
s, \quad c^{\prime}=c m, \quad H(c)=H^{\prime}(m c) .
\end{gathered}
$$

Also, the rate of fluid filtration everywhere below is considered positive constant and the strokes are omitted in the equations.

$$
\begin{gathered}
s_{t}=H(c)-s, \\
c_{t}-\Delta c+v \cdot \nabla c+s_{t}=0 .
\end{gathered}
$$

Beyond the change area of independent variables, the initial and boundary data old designations are preserved. Further, the problem $I^{\prime}\left(I^{\prime \prime}\right)$ is understood as task I (II) in which equations (1), (2) are replaced by (6), (7).

Theorem 1. Let the border $\Gamma \in 0^{2}$ function $u \in W_{q}^{2,1}\left(Q_{T}\right)$ conditions (3), (4) (respectively (3), (5)), $s_{0}, \quad c_{0}, \quad c_{\Gamma}(x, t)$ measurable and $0 \leq\left\{s_{0}(x), c_{0}, c_{\Gamma}(x, t)\right\} \leq$ $1, x \in \Omega$. Then the problem $I^{\prime}$ (accordingly the task $I I^{\prime}$ ) has a unique solution. In this case the following estimates are fair:

$$
\begin{array}{rr}
\|c\|_{q, Q_{T}}^{(2)} \leq K_{1}\left(1+\|u\|_{q, Q_{T}}^{(2)}\right), & 0 \leq \\
c(x, t) \leq 1 & (8) \\
0 \leq s(x, t) \leq 1, \quad & \left|s_{t}\right| \leq 1 .
\end{array}
$$

Positive constant $K_{1}$ depends only $q, \Omega$ and T. It should be noted, that when $q>(m+$ 2)/2 the solution $c \in H^{\alpha}\left(Q_{T}\right)$ for some $\alpha>$ 0 . When $q>m+2$ it becomes Holders and $\nabla c$.

4. Existence. The function $H(c)$ s approximated by continuous monotonic functions $\mathrm{H}_{n}(c)$

at $\quad c>\frac{1}{n}+c_{*}, c<c_{*}, n=1,2,3, \ldots$. Through $(6)_{n},(7)_{n}$ denoted the equation (6), (7), where instead of the function $\mathrm{H}$ function $\mathrm{H}_{n}$ is considered. For each $\mathrm{n}$ an approximate problem $(6)_{n},(7)_{n},(3)$, (4) is considered. The operator is determined $P: W_{q}^{2,1}\left(Q_{T}\right) \rightarrow$ $W_{q}^{2,1}\left(Q_{T}\right)$ fixed point which gives a solution to this problem. 
Let $c, g \in W_{q}^{2,1}\left(Q_{T}\right)$, then by definition $c=P(g)$, if $c$ satisfies the equation (7)

$$
\begin{aligned}
s(x, t)=s_{0}(x) & \cdot e^{-t} \\
& +\int_{0}^{t} H_{n}(g(x, \mu)) \cdot e^{\mu-t} d \mu
\end{aligned}
$$

and conditions (3), (4).

For $s(x, t)$, from the written submission, the assessment should be performed (9). Then for $c(x, t)$ assessment is performed (8). Conditions (3), (4), (8) determine in $W_{q}^{2,1}\left(Q_{T}\right)$ some convex, closed, bounded subset, which the operator $\mathrm{P}$ translates into itself. Since P is completely continuous, by the Schauder theorem there is a fixed point of the operator $\mathrm{P}$, which gives the solution to the problem $(6)_{n}$, $(7)_{n},(3),(4)$. We denote it by $\left\{c_{n}, s_{n}\right\}$. The second estimate in (8) follows from the maximum principle. In this to obtain the upper estimate, the cutting function is introduced: $\hat{c}=\max \{0, c-1\}$. To obtain a lower estimate, the cutting function has the form: $\hat{c}=\min \{0, c\}$. Estimates (8), (9) for $c_{n}, s_{n}$ and limitation $H_{n}\left(c_{n}\right)$ allow us to select subsequences $n_{k}$, such that:

$$
\begin{aligned}
c_{n_{k}} \rightarrow c \text { a.e. in } Q_{T}, \frac{\partial c_{n_{k}}}{\partial t} \rightarrow \frac{\partial c}{\partial t}, \quad \nabla c_{n_{k}} \rightarrow \\
\nabla c, \Delta c_{n_{k}} \rightarrow \Delta c \text { weakly in } L_{q}\left(Q_{T}\right), \\
s_{n_{k}} \rightarrow s, \frac{\partial s_{n_{k}}}{\partial t} \rightarrow \frac{\partial s}{\partial t}, \quad H_{n_{k}}\left(c_{n_{k}}\right) \rightarrow \mathrm{h} \\
\text { * weakly in } L_{\infty}\left(Q_{T}\right) .
\end{aligned}
$$

From the function definition $H_{n}\left(c_{n}\right)$ and $c_{n_{k}} \rightarrow c$ a.e. in $Q_{T}$, follows that $h(x, t)=H(c(x, t))$ a.e. in $Q_{T} \backslash E_{c}$. On the set $E_{c}$ function $h(x, t)=s(x, t)$ and by definition of function $H(c)$ and lemma 2 : $0 \leq h(x, t) \leq 1$. So on set $E_{c}$ function $h(x, t)$ coincides with $(c(x, t))$. Passing to the limit in (6) $)_{n},(7)_{n},(3),(4)$ at $n_{k} \rightarrow \infty$ we obtain, that the limit functions $c(x, t)$, $s(x, t)$ are the desired solution of the problem $I^{\prime}$.
5. Uniqueness. To prove the uniqueness the solvability of the adjoint problem is shown.

Let $c_{i}, s_{i}, i=1,2-$ two problem solutions $I^{\prime}$. We put

$$
\begin{gathered}
c=c_{1}-c_{2}, \quad s=s_{1}-s_{2}, \quad H= \\
H\left(c_{1}\right)-H\left(c_{2}\right) .
\end{gathered}
$$

Functions $c, s, H$ satisfy equations (6), (7) and conditions:

$$
c,\left.s\right|_{t=0}=0,\left.\quad c\right|_{\Gamma_{T}}=0,\left.\quad H\right|_{E}=s
$$

where $\quad E=E_{c_{1}} \cap E_{c_{2}} . \quad$ Auxiliary functions are introduced $F_{\delta}$ и $F_{\varepsilon, \delta}: F_{\delta}=H / c$ on set $\mathrm{E}=\left\{(\mathrm{x}, \mathrm{t}) \in Q_{T}|| c(x, t) \mid \geq \delta\right\} \quad$ and $F_{\delta}=0$ on $Q_{T} \backslash E$, and the function $F_{\varepsilon, \delta}$ is selected from the conditions:

$$
\begin{gathered}
F_{\varepsilon, \delta} \in \dot{\mathrm{C}}^{\infty}\left(Q_{T}\right), \quad 0 \leq F_{\varepsilon, \delta} \leq \\
F_{\delta}, \quad \lim _{\varepsilon \rightarrow 0}\left\|F_{\varepsilon, \delta}-F_{\delta}\right\|_{1, Q_{T}}=0 .
\end{gathered}
$$

In $Q_{T}$ functions $\varphi, \psi$ are considered, fairly smooth, satisfying the conditions:

$$
\begin{gathered}
\varphi,\left.\psi\right|_{t \in\left[T_{0}, T\right]}=0,\left.\quad \varphi\right|_{\Gamma_{T}}=0, \quad 0< \\
T_{0} \leq T
\end{gathered}
$$

From (6), (7), (10, (11), the equality is derived:

$$
\begin{gathered}
\int_{Q_{T}}\left\{c \cdot M_{1}(\varphi, \psi)+s \cdot M_{2}(\varphi, \psi)+\right. \\
\left.\left(H-F_{\varepsilon, \delta} c\right) \cdot \psi\right\} d x d t=0
\end{gathered}
$$

where $\quad M_{1}(\varphi, \psi)=\varphi_{t}+\Delta \varphi+v$. $\nabla \varphi+F_{\varepsilon, \delta} \psi, M_{2}(\varphi, \psi)=\varphi_{t}+\psi_{t}-\psi$.

Let be $G_{1}, G_{2} \in \dot{C}^{\infty}\left(Q_{T_{0}}\right)$ and in $Q_{T_{0}}$ equations are considered

$$
M_{i}(\varphi, \psi)=G_{i}, \quad i=1,2
$$

Solvability of problem (11), (13) is obtained in the standard way, based on the local existence theorem and a priori estimates. From (13) view is displayed for $\psi$ :

$$
\begin{gathered}
\psi(x, t)=\int_{t}^{T_{0}}\left\{\psi(x, \mu)+G_{2}(x, \mu)\right\} d \mu- \\
\varphi(x, t)
\end{gathered}
$$


Function $\Phi(\mathrm{x}, \mathrm{t})$ determined by the equality:

$$
\Phi(\mathrm{x}, \mathrm{t})=\varphi(x, t) \cdot e^{t}
$$

The following equality is a consequence of (13)-(15):

$$
\begin{array}{r}
\Phi \cdot \Phi_{t}+\Phi \cdot \Delta \Phi+v \cdot \Phi \cdot \nabla \Phi-\left(1+F_{\varepsilon, \delta}\right) \cdot \\
\Phi^{2}=\left\{G_{1}-F_{\varepsilon, \delta} \cdot \int_{t}^{T_{0}}\left(\psi+G_{2}\right) d \mu\right\} \cdot e^{t} \cdot \Phi
\end{array}
$$

Consider (16) at the point of the internal maximum of the function $\Phi$. The first two terms at this point are nonpositive and the third term turn to zero, so taking into account (15), we obtain the estimate:

$$
\begin{gathered}
|\varphi| \leq|\Phi| \leq\left\{\int_{0}^{T_{0}}\left(|\psi|+\left|G_{2}\right|\right) d t+\left|G_{1}\right|\right\} . \\
e^{T_{0}}
\end{gathered}
$$

From (14) and (17) using the Gromwell inequality, the following estimate is obtained:

$$
\|\psi\|_{\infty, Q_{T}} \leq e^{T_{0}}\left\|G_{1}\right\|_{\infty, Q_{T_{0}}}+K_{2} \cdot T_{0}
$$

Constant $K_{2}$ depends on $\mathrm{T}$ and norms $\left\|G_{i}\right\|_{\infty, Q_{T_{0}}}, \mathrm{i}=1,2$. After the passage to the limit $\rightarrow 0$, then by $\delta \rightarrow 0$ by virtue of estimate (18) and conditions (10), equality (12) takes the form:

$\int_{Q}\left\{c \cdot G_{1}+s \cdot G_{2}\right\} d x d t+\int_{E} \psi \cdot s d x d t=$ 0.

We can assume that $G_{i}, i=1,2-$ arbitrary functions from $L_{\infty}\left(Q_{T_{0}}\right)$. Suppose $T_{0}=\min (\mathrm{T}$,

$\left.\frac{1}{2 \cdot K}\right)$. Choosing $G_{1}=0, G_{2}=\operatorname{sign} s$, from (18), (19) we get, that $s=0$ in $Q_{T_{0}}$. At $G_{2}=$ $0, G_{1}=$ sign $\mathrm{c}$ from (19) $\mathrm{c}=0$ in $Q_{T_{0}}$. Similarly, uniqueness is shown in $\Omega x\left[T_{0}\right.$, $\left.2 \cdot T_{0}\right], \Omega x\left[2 \cdot T_{0}, 3 \cdot T_{0}\right]$ etc. For a finite number of steps, we obtain uniqueness in $Q_{T}$.
IV. THE CONTINUITY OF THE SOLUTION ON THE INITIAL AND BOUNDARY DATA.

Let be $c_{i}, s_{i}, i=1,2-$ two solutions of the problem $I I^{\prime}$, satisfying the initial and boundary conditions:

$$
\begin{gathered}
c_{i}(x, 0)=u_{i}(x, 0), s_{i}(x, 0) \\
=s_{0 i}(x), \quad x \in \Omega . \\
\left.\frac{\partial c_{i}}{\partial n}\right|_{\Gamma_{T}}=\left.\frac{\partial u_{i}}{\partial n}\right|_{\Gamma_{T}}, \quad i=1,2 .
\end{gathered}
$$

We introduce the notation:

$$
\begin{gathered}
c=c_{1}-c_{2}, \quad s=s_{1}-s_{2}, \quad H \\
=H\left(c_{1}\right)-H\left(c_{2}\right), \quad s_{0} \\
=s_{01}-s_{02}, \\
u=u_{1}-u_{2}, \quad \delta=\|u\|_{q, Q_{T}}^{(2)}+ \\
\left\|s_{0}\right\|_{\infty, \Omega}, \quad c_{0}=c_{01}-c_{02}, \quad c_{\Gamma}=c_{\Gamma 1}- \\
c_{\Gamma 2} .
\end{gathered}
$$

Then the functions c, s, $\mathrm{H}$ satisfy equations (6), (7) and conditions (3), (5).

Theorem 2. If the assumptions of Theorem 1 are fulfilled for the functions $u_{i}, s_{0 i}, i=1,2$ then the following estimates are true:

$$
\|c\|_{q, Q_{T}}^{(2)} \leq K_{3} \cdot \delta^{1 / q}
$$

$$
\left\|s_{t}\right\|_{p, Q_{T}}+\|s\|_{p, Q_{T}} \leq K_{4} \cdot \delta^{1 / p}
$$

where $1 \leq p<\infty$, constants $K_{3}, \quad K_{4}$ depend on $q, T, \Omega,\left\|u_{i}\right\|_{q, Q_{T}}^{(2)},\left\|s_{0 i}\right\|_{\infty, \Omega}, i=$ 1,2 .

Proof. Multiply equation (7) by $c$. $\left(c^{2}+\varepsilon\right)^{-\frac{1}{2}}, \varepsilon>0$, and integrate over the region $Q_{\theta}$ :

$$
\begin{aligned}
& \int_{Q_{\theta}}\left\{\left[\left(c^{2}+\varepsilon\right)^{\frac{1}{2}}\right]_{t}+\varepsilon \cdot(\nabla c)^{2} \cdot\left(c^{2}+\varepsilon\right)^{-\frac{3}{2}}+\right. \\
& \left.H \cdot c \cdot\left(c^{2}+\varepsilon\right)^{-\frac{1}{2}}-\mathrm{s} \cdot c \cdot\left(c^{2}+\varepsilon\right)^{-\frac{1}{2}}\right\} d x d t= \\
& =\int_{\Gamma} \mathrm{c}_{\Gamma} \cdot \mathrm{c} \cdot\left(\mathrm{c}^{2}+\varepsilon\right)^{-\frac{1}{2}} d \Gamma+\varepsilon \cdot v \cdot \\
& \int_{Q_{\theta}} \nabla\left[\left(c^{2}+\varepsilon\right)^{\frac{1}{2}}\right] d x d t .
\end{aligned}
$$


Drop the nonnegative term in this equality $\varepsilon \cdot(\nabla c)^{2} \cdot\left(c^{2}+\varepsilon\right)^{-\frac{3}{2}}$ and in the resulting inequality we go to the limit by $\varepsilon \rightarrow 0$ :

$$
\begin{array}{r}
\int_{\boldsymbol{Q}_{\boldsymbol{\theta}}}\left\{|\boldsymbol{c}|_{\boldsymbol{t}}+|H|-|\mathrm{s}|\right\} d x d t \leq \int_{\Gamma}\left|\mathrm{c}_{\Gamma}\right| d \Gamma \\
\int_{\boldsymbol{Q}_{\boldsymbol{\theta}}}\left\{|\boldsymbol{s}|_{\boldsymbol{t}}+|\mathrm{s}|-|H|\right\} d x d t \leq 0 .
\end{array}
$$

From inequalities (22), (23) follows that

$$
\begin{gathered}
\int_{\Omega}\{|c(x, \theta)|+|s(x, \theta)|\} d x \leq\left\|c_{0}\right\|_{1, \Omega}+ \\
\left\|s_{0}\right\|_{1, \Omega}+\left\|c_{\Gamma}\right\|_{1, \Gamma_{\theta}} \leq \mathrm{K} \cdot \delta .
\end{gathered}
$$

From the estimates (22), (24) the inequality is derived: $\|H\|_{1, Q_{T}} \leq K \cdot \delta$. From here and from equation (6) is estimated: $\left\|s_{t}\right\|_{1, Q_{T}} \leq$ $K \cdot \delta$. Evaluation (21) is a consequence of the resulting assessment and the limitations of the norm $\|s\|_{\infty, Q_{T}}$. The considered function $\mathrm{c}(\mathrm{x}, \mathrm{t})$, as a solution to problem (7), (3), (5) and, taking into account (21), we obtain the estimate (20).

It should be noted, to get ordinary continuity you need to show that $\lim _{\delta \rightarrow 0}\left\|s_{t}\right\|_{\infty, Q_{T}}=0$. In general, this equality does not performed.

Suppose we have two solutions to the problem under consideration: $\mathrm{c} \equiv 0, s \equiv 0$ и $c(x, t)=1-s(x, t)$,

$0<\varepsilon<1, \quad s(x, t)=$ $\begin{cases}1-e^{-t}, & 0 \leq t \leq-\ln (1-\varepsilon), \\ \varepsilon, & -\ln (1-\varepsilon) \leq t \leq T .\end{cases}$

The second solution satisfies the following initial and boundary data:

$$
\begin{gathered}
\left.c\right|_{t=0}=\varepsilon,\left.\quad s\right|_{t=0}=0,\left.\quad\left(\frac{\partial c}{\partial n}-v \cdot c\right)\right|_{\Gamma_{T}} \\
=0 .
\end{gathered}
$$

Obviously, in this case $\delta=\varepsilon$, a $\left\|s_{t}\right\|_{\infty, Q_{T}}=1$.
1. Limit transation in relaxation time. Below, we will study the behavior of the solution as $\tau \rightarrow 0$ using the example of problem 1. For problem II a similar result is true. We introduce $K\left(Q_{T}\right)$ - function space with the norm:

$$
\begin{gathered}
\|u\|_{K, T}=\|u\|_{\infty, Q_{T}}+\left\|u_{t t}\right\|_{1, Q_{T}}+ \\
\|\nabla u\|_{2, Q_{T}}+\left\|u_{t}\right\|_{2, Q_{T}}+\left\|\nabla u_{t}\right\|_{2,1, Q_{T}}
\end{gathered}
$$

Functions $\quad c^{\tau}(x, t), \quad s^{\tau}(x, t) \quad$ satisfying equations (1), (2) and conditions are considered:

$\left.\left(c^{\tau}(x, t)-c_{0}^{\tau}\right)\right|_{\Gamma_{\mathrm{T}} \cup\{t=0\}}=0,\left.\quad s^{\tau}\right|_{t=0}=s_{0}^{\tau}$

where $c_{0}^{\tau} \in W_{q}^{2,1}\left(Q_{T}\right) \cap K\left(Q_{T}\right), \quad s_{0}^{\tau} \in$ $L_{\infty}(\Omega)$ and $s_{0}^{\tau}(x)=H\left(c_{0}^{\tau}(x)\right), \quad x \in \Omega$.

Without loss of generality, constants everywhere below are considered, equal to one.

Lemma 3. To solve problem (1), (2), (25), (26), the following estimates are valid.:

$$
\left\|c^{\tau}\right\|_{\infty, Q_{T}} \leq K_{5},
$$

$$
\left\|c_{t}^{\tau}\right\|_{2, Q_{T}}+\max _{[0, T]}\left\|\nabla c^{\tau}\right\|_{2, \Omega} \leq K_{6},
$$

$$
\left\|H\left(c^{\tau}\right)-s^{\tau}\right\|_{1, Q_{T}^{\delta}} \leq K_{7} \cdot \delta^{-1 / 2} \cdot \tau, \quad \delta>0
$$

where $\quad Q_{T}^{\delta}=\Omega^{\delta} x(0, T), \quad \Omega^{\delta}=$ $\{x \in \Omega|\operatorname{dist}|(x, \mu)>\delta\}, \delta>0\}$, and the constants $K_{i}, \mathrm{i}=5,6,7$ depend only on $T, \Omega$ and $\left\|c_{0}^{\tau}\right\|_{K, T}$.

Proof. From equation (1), (2) and condition (26) we get follow estimates:

$$
\begin{array}{r}
0 \leq s^{\tau}(x, t) \leq 1, \quad s_{t}^{\tau} \cdot\left(c^{\tau}-c^{*}\right) \geq \\
0, \quad \int_{0}^{\theta} s_{t}^{\tau} \cdot\left(c^{\tau}-c^{*}\right){ }_{t} d t \geq 0, \theta \leq \mathrm{T} .
\end{array}
$$

From the maximum principle should also be estimated: $0 \leq \mathrm{c}^{\tau}(x, t) \leq 1$. 
Here and further, where there is no misunderstanding, the index $\tau$ of the functions $c_{0}^{\tau}, c^{\tau}, s^{\tau}$-is omitted.

Let us show how the last one turns out:

$$
\begin{aligned}
& \tau \int_{0}^{\theta} s_{t} \cdot\left(c-c^{*}\right)_{t} d t=\int_{0}^{\theta}(H(c)-s) \cdot \\
& \left(c-c^{*}\right)_{t} d t=\left.(H(c)-s) \cdot\left(c-c^{*}\right)\right|_{0} ^{\theta}+ \\
& +\int_{0}^{\theta} s_{t} \cdot\left(c-c^{*}\right) d t \geq 0 .
\end{aligned}
$$

From the resulting estimates and on the basis of the results from [2] the estimate (28) follows. To obtain the estimate (29), the function is introduced:

$$
\begin{gathered}
f \in \\
W_{2}^{1}(\Omega),\left.\quad f\right|_{\Omega^{\delta}}=1, \quad\|\dot{\nabla} f\|_{2, \Omega} \leq K(\Omega) \cdot \delta^{-1 / 2}
\end{gathered}
$$

Further, equation (2) is multiplied by $f \cdot c$. $\left(c^{2}+\varepsilon\right)^{-\frac{1}{2}}, \quad \varepsilon>0$ and integrated by $Q_{T}$ :

$$
\begin{aligned}
& \int_{Q_{\theta}}\left\{\left[f \cdot\left(c^{2}+\varepsilon\right)^{\frac{1}{2}}\right]_{t}+\varepsilon \cdot f \cdot(\nabla c)^{2} .\right. \\
& \left.\left(c^{2}+\varepsilon\right)^{-\frac{3}{2}}+s_{t} \cdot f \cdot c \cdot\left(c^{2}+\varepsilon\right)^{-\frac{1}{2}}\right\} d x d t= \\
& =\varepsilon \cdot v \cdot \int_{Q_{\theta}} \nabla f \cdot \nabla\left[\left(c^{2}+\varepsilon\right)^{\frac{1}{2}}\right] d x d t+ \\
& \int_{Q_{\theta}} \nabla f \cdot \nabla c \cdot c \cdot\left(c^{2}+\varepsilon\right)^{-\frac{1}{2}} d x d t .
\end{aligned}
$$

Passing to the limit at $\varepsilon \rightarrow 0$ and discarding the nonnegative terms in the left-hand side, we obtain the estimate (29). The lemma is proved.

Let be $U=c+H(c)$ generalized solution of the Stefan problem (see definition, for example, in [9]), satisfying initial and boundary conditions:

$$
\begin{array}{r}
U(x, 0)=c_{0}(x, 0)+ \\
s_{0}(x), \quad x \in \Omega,
\end{array}
$$

$$
c(x, t)=c_{0}(x, t), \quad(x, t) \in \Gamma_{T},
$$

where $\quad \mathrm{c}_{0} \in K\left(Q_{T}\right), \quad s_{0} \in L_{\infty}(\Omega), \quad s_{0}=$ $H\left(c_{0}(x, 0)\right)$.

\section{Teorem 3. If}

$$
\left\|\mathrm{c}_{0}^{\tau}-\mathrm{c}_{0}\right\|_{K, T}+\left\|s_{0}^{\tau}-s_{0}\right\|_{1, \Omega} \rightarrow 0 \text { at } \begin{gathered}
\tau \rightarrow \\
0,
\end{gathered}
$$

then $\mathrm{c}^{\tau}+s^{\tau}$ converges to $\mathrm{U}$ at $\tau \rightarrow 0$ in the following sense:

$$
\begin{aligned}
& c^{\tau_{k}} \rightarrow c^{\circ} \text { weakly in } W_{2}^{1}\left(Q_{T}\right), \quad * \text { - weakly } \\
& \text { in } L_{\infty}\left(Q_{T}\right) \text {, } \\
& s^{\tau_{k}} \rightarrow H\left(c^{\circ}\right) \quad * \text { - weakly in } \\
& L_{\infty}\left(Q_{T}\right) \text { at } \tau_{k} \rightarrow 0
\end{aligned}
$$

Really, the functions $c^{\tau}, s^{\tau}$ satisfy the identity::

$$
\begin{aligned}
\int_{Q_{T}}\left\{\mathrm{c}^{\tau} \cdot \varphi-\left(\mathrm{c}^{\tau}+s^{\tau}\right) \cdot \varphi_{t}\right\} d x d t \\
\quad=\int_{\Omega}\left(c_{0}^{\tau}+s_{0}^{\tau}\right) \cdot \varphi(x, 0) d x
\end{aligned}
$$

for any $\varphi \in W_{2}^{1}\left(Q_{T}\right),\left.\quad \varphi\right|_{\Gamma_{T} \cup\{t=0\}}=0$. Then, from conditions (31) and estimates (27) - (29) we can choose a subsequence that , $\tau_{k} \rightarrow 0$ (32) are fulfilled due to the uniqueness of the whole family converges to solving the Stefan problem as $\tau \rightarrow 0$.

\section{NUMERICAL EXPERIMENTS.}

Before proceeding to the construction of algorithms for solving the original problem, we will analyze one model problem from [5], which has important practical significance, since it is the basis of the models, taking into account the given equations of the kinetics of changes in any parame.

$$
\frac{\partial u}{\partial t}-\Delta u+\chi \frac{\partial w}{\partial t}=0
$$

$$
\frac{\partial w}{\partial t}=\frac{1}{\tau}[H(u)-w]+\lambda \Delta w
$$




$$
H(u)=\left\{\begin{array}{l}
1, u>u^{*} \\
w, u=u^{*} \\
0, u<u^{*}
\end{array}\right.
$$

where $H(u)$ characterizes the phase transition, $\tau$ - relaxation time, $\chi, \lambda$ positive weight coefficients, taking into account the nature of the contribution process (transience and slowness).

For (33) - (35) the following initial and boundary conditions are given:

$$
\begin{aligned}
& u(x, 0)=u_{0}(x), w(x, 0)=w_{0}(x), \\
& x \in \Omega
\end{aligned}
$$

$$
u(x, t)=u_{s}(x, t), \quad(x, t) \in S_{T}
$$

The correctness of the problem (33) (37) and the qualitative properties of the solutions (the asymptotic behavior of the solution with an unlimited increase in time, limiting transitions in relaxation time and $\lambda$ - migration of moisture It is proved that problem (33) - (37) is a problem of Stefan type. When $\lambda \rightarrow 0$, problem (33) - (37) tends to solve the problem without taking into account moisture migration.) were investigated in detail in [5].

It is known, that systems of equations like (33) - (37) have a number of features, in particular, depending on the values $\tau, \chi$,

$\lambda$ and $u^{*}$ the solution of the system is either completely absent, or contains jumps, which leads to the expansion or contraction of the phase transition zone. We present a numerical algorithm only for the onedimensional case with a given type of boundary and initial data, i.e.:

$$
u_{t}-u_{x x}+\chi w_{t}=0
$$

$$
\begin{aligned}
& w_{t}=\frac{1}{\tau}[H(u)-w]+\lambda w_{x x} \\
& H(u)=\left\{\begin{array}{l}
1, u>u^{*} \\
w, u=u^{*} \\
0, u<u^{*}
\end{array}\right.
\end{aligned}
$$

initial and boundary conditions:

$$
\begin{gathered}
u(x, 0)=e^{-x}, \\
w(x, 0)=1-e^{-x}=w_{0}(x), x \in \Omega \\
u(0, t)=(1+t)^{2}, u(1, t)=(1+t)^{3}, \\
(x, t) \in S_{T}
\end{gathered}
$$

In system (38) - (42) at the beginning we solve (39) with the corresponding boundary conditions, for a given value $u$. Further we find $u$ at the found value of $w$. To do this, we introduce a uniform grid $x_{i}=x_{0}+i h, i=\overline{0, N}$, where $h=$ const $\quad-\quad$ grid spacing $t^{n}=n \tilde{\tau}, n=0,1,2 \ldots$, here $\tilde{\tau}$ - time step. Required functions $u, w$ at the nodes $\left(x_{i}, t^{n}\right)$ hereinafter referred to as $u_{i}^{n}, w_{i}^{n}$ respectively. Then we write the difference form of the equation of system (38) and (39) for a constant grid step $h$ :

$$
\begin{aligned}
& \frac{1}{\tau}\left(H\left(u_{i}^{n}\right)-w_{i}^{n+1}\right)+\lambda \frac{w_{i+1}^{n+1}-2 w_{i}^{n+1}+w_{i-1}^{n+1}}{h^{2}}=\frac{w_{i}^{n+1}-w_{i}^{n}}{\tilde{\tau}} \\
& \frac{u_{i}^{n+1}-u_{i}^{n}}{\tau}+\chi \frac{w_{i}^{n+1}-w_{i}^{n}}{\tilde{\tau}}=\frac{u_{i+1}^{n+1}-2 u_{i}^{n+1}+u_{i-1}^{n+1}}{h^{2}}
\end{aligned}
$$

This system is solved sequentially by applying the sweep method to each equation. The stability conditions of the sweep method are satisfied. 


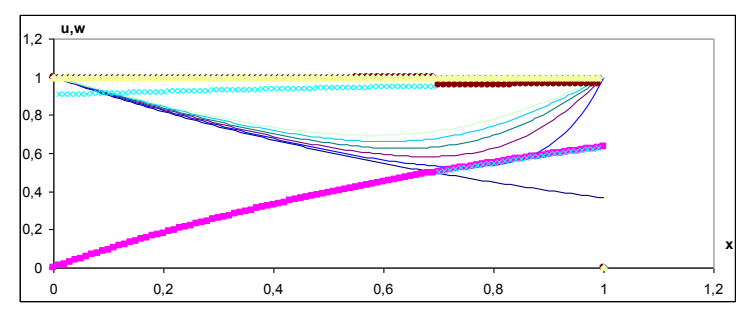

Рисунок 1 - Распределение $u$ (тонкая линия) и $w$ (толстая линия)

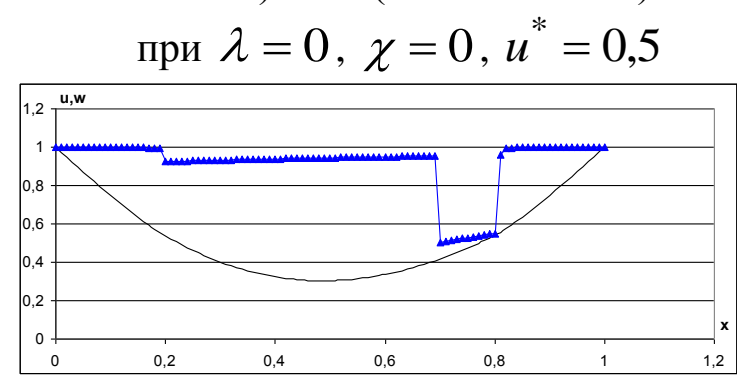

Picture 2 - Distribution $u$ (thin line) and $w$ (thick line)

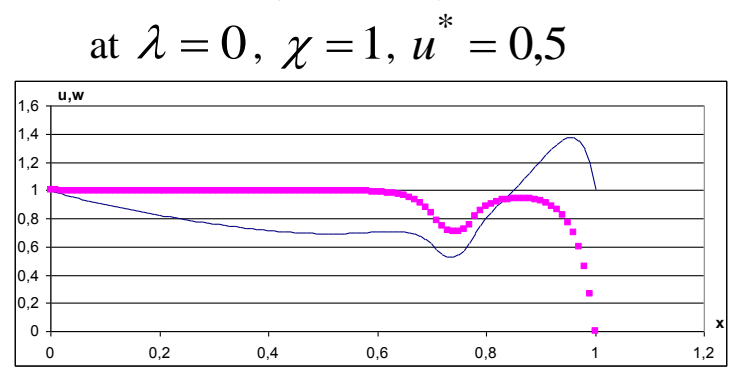

Picture 3 - Distribution $u$ (thin line) and $w$ (thick line)

at $\lambda=1, \chi=0, u^{*}=0,5$

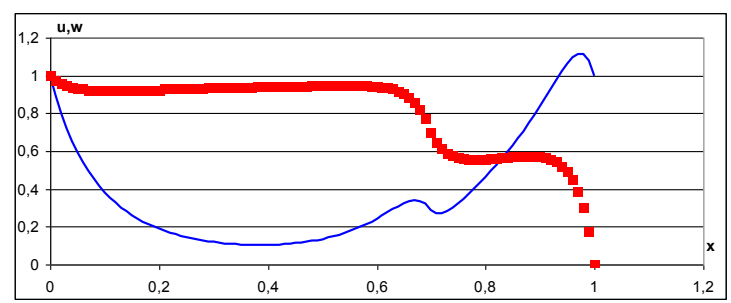

Picture 4 - Distribution $u$ (thin line) and $w$ (thick line)

at $\lambda=1, \chi=1, u^{*}=0,5$

The obtained numerical results take place for solving the original problem after simple transformations. In addition, the results of [5] are valid for solving the original problem.

\section{REFERENCES}

1. Kaliev I.A., Mukhambetzhanov S.T., Sabitova G.S. Numerical modeling of the non-equilibrium sorption process // Ufa Mathematical Journal. - 2016. - Vol. 8 (2). - P. 39-43.

2. Kaliev I.A., Rasinkov E.N. About the Stefan problem with phase relaxation // Collection of scientific papers DCC, Edition. 91, 1989, p. 21-36

3. Lapidus L., Amundson W.R. Mathematics of adsorption in beds. VI. The effect of longitudinal diffusion in ion exchange and chromatographic columns // J.Phys. Chem. 1952. V.56. P. 984-988.

4. Kaliev I.A., Sabitova G.S. On a problem of nonequilibrium sorption // Journal of Applied and Industrial Mathematics. 2003. Vol. VI, №1 (13). P. 35-39

5. Kaliev I.A., Mukhambetzhanov S.T., Rasinkov E.N. The correctness of the mathematical model of nonequilibrium phase transitions of water in porous media // Collection Dynamics of a continuous medium. Institute of Hydrodynamics named after MA Lavrentyev, Siberian Branch of the Russian Academy of Sciences. Novosibirsk. Edition №93,94,1989г., p.4659.

6. Ahmed-Zaki D.Zh., Mukhambetzhanov S.T., Imankulov T.S. Design of i-fields system component: Computer model of oilrecovery by polymer flooding // Informatics in Control, Automation and Robotics (ICINCO), 12th International Conference. 2015. - Vol. 2. - P. 510-516.

7. Meirmanov A.M., Mukhambetzhanov S.T., Nurtas, M. Seismic in composite media: Elastic and poroelastic components// Siberian Electronic Mathematical Reports. 2016.

8. Kenzhebayev T.S., Mukhambetzhanov S.T. Numerical Solution Of The Inverse Problem Of Filtration Theory By Modulating Functions// Far East Journal of Mathematical Sciences. - 2016. - Vol. 99 (12). - P. 1779

9. Мейрманов A.M. Stefan problem. Novosibirsk.: Science, 1986. -239c.

10. Coats K.H., Smith B.D. Dead and pore volume and dispersion in porous media // Soc. Petrol. Eng. J. 1964. V. 4, N 1. P. 73-84 
11. Meirmanov A.M., Mukhambetzhanov S.T., Nurtas, M. Seismic in composite media: Elastic and poroelastic components// Siberian Electronic Mathematical Reports. 2016.

12. Kenzhebayev T.S., Mukhambetzhanov S.T. Numerical Solution Of The Inverse Problem Of Filtration Theory By Modulating Functions// Far East Journal of Mathematical Sciences. - 2016. - Vol. 99 (12). - P. 1779 